\title{
Local Kinetic Effects in Two-Dimensional Plasma Turbulence
}

\author{
S. Servidio, ${ }^{1}$ F. Valentini, ${ }^{1}$ F. Califano, ${ }^{2}$ and P. Veltri ${ }^{1}$ \\ ${ }^{1}$ Dipartimento di Fisica, Università della Calabria, I-87036 Cosenza, Italy \\ ${ }^{2}$ Dipartimento di Fisica and CNISM, Università di Pisa, 56127 Pisa, Italy
}

(Received 28 July 2011; published 26 January 2012)

\begin{abstract}
Using direct numerical simulations of a hybrid Vlasov-Maxwell model, kinetic processes are investigated in a two-dimensional turbulent plasma. In the turbulent regime, kinetic effects manifest through a deformation of the ion distribution function. These patterns of non-Maxwellian features are concentrated in space nearby regions of strong magnetic activity: the distribution function is modulated by the magnetic topology, and can elongate along or across the local magnetic field. These results open a new path on the study of kinetic processes such as heating, particle acceleration, and temperature anisotropy, commonly observed in astrophysical and laboratory plasmas.
\end{abstract}

PACS numbers: 52.35.Ra, 52.65.-y, 94.05.-a, 96.50.Ci

Turbulence, a complex problem in fluid dynamics, is an even more challenging subject in a plasma, since crossscale couplings and kinetic effects are present [1]. In a collisional (fluid) description of plasmas, regions in between coherent structures are expected to be sites of enhanced dissipation, where processes such as magnetic reconnection and plasma heating may be at work [1]. On the other hand, in collisionless plasmas, as, for example, the solar wind, kinetic processes may lead to phenomena such as temperature anisotropy, heating, particle energization, entropy cascade, and so on [1-4]. A robust and quantitative description of these nonlinear features, commonly observed in nature, has not been proposed yet.

Kinetic turbulence in plasmas represents an unresolved problem, and analytical treatments such as linear and quasilinear simplifications of the Vlasov-Maxwell equations may be of some help [5]. On the other hand, since plasmas are commonly subject to turbulence, the above simplified models may fail to provide a valid description. In general, a valid support is given by kinetic direct numerical simulations, in which the time evolution of the particles distribution function (hereafter DF) is described self-consistently. In turbulent systems such as the solar wind [6,7], for example, it is of crucial relevance to quantify the role of kinetic effects in the turbulent cascade, since they may explain the energy dissipation mechanisms. NonMaxwellian features of the DF represent one of the most direct manifestations of these complex kinetic processes.

In simplified kinetic descriptions of the plasma, it is expected that the DF deforms manifesting enhanced temperature in the direction parallel (or antiparallel) to the global mean magnetic field. This may be due, for example, to resonant ion-cyclotron interaction [2,8] or to the generation of field-aligned beams [9]. It is not trivial that the above statements still hold in a fully turbulent regime. In turbulence, moreover, one may ask if kinetic effects are homogeneous in space, or if they are concentrated in certain regions of the turbulent field-in the sense that they manifest as spatial patches. The scenario can also depend on parameters such as the system size or the level of turbulence. In the present work we provide some answers to the above questions.

In this Letter we propose an alternative point of view on the description of plasma turbulence, showing that the ion DF is modulated by the local mean magnetic field, in a complex way. We solve numerically the hybrid VlasovMaxwell system, using a Eulerian algorithm [10], in a 2D-3V geometry (two dimensions in physical space and three in velocity space), to investigate the link between spatial magnetic structures, such as magnetic vortices, and the formation of non-Maxwellian features. The latter will be quantified computing moments of the DF, at each position of the turbulent field. A statistical description of the link between the magnetic skeleton of turbulence and the velocity subspace of the DF will be presented.

The dimensionless hybrid Vlasov-Maxwell equations (kinetic ions and fluid electrons) are given by [10]

$$
\begin{aligned}
& \partial_{t} f+\boldsymbol{\nabla} \cdot(\boldsymbol{v} f)+\nabla_{v} \cdot[(\boldsymbol{E}+\boldsymbol{v} \times \boldsymbol{B}) f]=0, \\
& \boldsymbol{E}=-\boldsymbol{u} \times \boldsymbol{B}+\boldsymbol{j} \times \boldsymbol{B} / n-\boldsymbol{\nabla} P_{e} / n+\eta \boldsymbol{j},
\end{aligned}
$$

where $f(\boldsymbol{x}, \boldsymbol{v}) \equiv f\left(x, y, \boldsymbol{v}_{x}, \boldsymbol{v}_{y}, \boldsymbol{v}_{z}\right)$ is the ion distribution function, $\boldsymbol{E}$ the electric field, $\boldsymbol{B}=\boldsymbol{b}+\boldsymbol{B}_{0}$ the total magnetic field $\left(\boldsymbol{B}_{0}=B_{0} \hat{z}\right.$ is the mean field), and $\boldsymbol{j}=\boldsymbol{\nabla} \times \boldsymbol{b}$ the total current density. The ion density $n$ and the ion bulk velocity $\boldsymbol{u}$ are obtained as the velocity moments of $f$, while an isothermal equation of state for the electron pressure $P_{e}$ has been assigned. In Eq. (1) times are scaled by the cyclotron time $\Omega_{c i}^{-1}$, velocities by the Alfvén speed $V_{A}$, lengths by the ion skin depth $d_{i}=V_{A} / \Omega_{c i}$, and masses by the ion mass $m_{i}$. In order to suppress spurious numerical effects due to the presence of strong current sheets, a resistive term in the Ohm's law has been added as a standard (numerical) Laplacian dissipation. The resistivity $\eta$ is given small values to achieve both high Reynolds numbers and to ensure adequate spatial resolution 
( $\eta \sim 10^{-2}$ for the runs reported here). The latter choice is not intended to mimic any specific plasma kinetic process, but rather to damp out numerical instabilities that may strongly damage the genuine properties of small-scale turbulence [11]. Electron inertia effects have not been included in Eq. (1) [12].

The initial condition consists of a Maxwellian plasma perturbed by a 2D spectrum of Fourier modes, imposed for both the velocity and the magnetic field. To avoid an artificial compressive activity, neither density perturbations nor parallel variance $\left(b_{z}, v_{z}\right)$ are imposed at $t=0$. Energy has been injected, with random phases, in the range $2 \leq m \leq 6$, where $m=\left(L_{0} / 2 \pi\right) k$, and $L_{0}=2 \pi \alpha d_{i}$ being the system size ( $\alpha$ is a positive real number). Periodic boundary conditions have been employed. The ion plasma beta is $\beta=2 v_{t i}^{2} / V_{A}^{2}=2\left(v_{t i}\right.$ is the ion thermal speed), while the electron to ion temperature ratio is fixed at $T_{e} / T_{i}=1$. The limits of the velocity domain in each direction are fixed at $v^{\max }= \pm 5 v_{t i}$. For all runs, $512^{2}$ mesh points in physical space and $51^{3}$ in velocity space are used. To investigate the influence of both turbulence and system size, we performed different runs varying $\delta b / B_{0}\left(\delta b=\left\langle b_{x}^{2}+b_{y}^{2}\right\rangle\right.$ and $\langle\bullet\rangle$ represents spatial averages) and $L_{0} / d_{i}$. Simulations are reported in Table I.

In analogy with fluid models, in decaying turbulence there is an instant of time, let us say $\tau^{\star}$, at which the turbulent activity is maximum [13]. This time can be estimated measuring the average out-of-plane squared current density $\left\langle j_{z}^{2}\right\rangle$. At $\tau^{\star}$, summarized for each run in Table I, we perform our analysis (a study of the time evolution will be presented in future works.) As follows, we give a brief overview on turbulence, analyzing Run II (all the simulations give qualitatively similar results). As represented in Fig. 1(a), turbulence manifests through the appearance of coherent structures, exhibiting a sea of vortices (islands) and current sheets. This can be seen in contour maps of $j_{z}$ and $a_{z}$, where $a_{z}$ is the magnetic potential of the inplane magnetic field $\boldsymbol{b}_{\perp}=\boldsymbol{\nabla} a_{z} \times \hat{z}$. In between islands $j_{z}$ becomes very intense, being a signature of the intermittent nature of the magnetic field [6]. In these regions of high magnetic stress, reconnection locally occurs at the $X$ points of $a_{z}$ [crosses in Fig. 1(a)] [14,15]. From a qualitative analysis, the size of these current sheets is of the order of few $d_{i}$ 's (note that these also manifest a bifurcation, typical signature of the Hall effect).

To quantify turbulence, we computed the power spectra for the density $n$, the ion bulk velocity $\boldsymbol{u}$, the magnetic $\boldsymbol{b}$,

TABLE I. Initial amount of magnetic fluctuations (second column), system size (third column), and time of the peak of the turbulent activity (last column).

\begin{tabular}{lccc}
\hline \hline & $\delta b / B_{0}$ & $L_{0} / d_{i}$ & $\tau^{\star}\left(\Omega_{c i}^{-1}\right)$ \\
\hline Run I & $1 / 7$ & $2 \pi \times 30$ & 150 \\
Run II & $1 / 3$ & $2 \pi \times 20$ & 50 \\
Run III & $1 / 3$ & $2 \pi \times 10$ & 20 \\
\hline \hline
\end{tabular}

and electric $\boldsymbol{E}$ fields. These power spectra reveal several features commonly observed in space plasmas and that are shown in Fig. 1(b). As observed in solar wind turbulence [6], the large scale activity is essentially incompressible, namely $\left|n_{k}\right|^{2}$ is negligible for low $k$ 's [6]. The Alfvénic correlation between the magnetic and the velocity field, typical of magnetohydrodynamic turbulence, is broken at $k d_{i} \sim 1[10,16]$. At small scales, comparable or smaller than $d_{i}$, the spectra become steeper, due mainly to the presence of kinetic effects. As in previous works $[7,10,16]$, it is worth noting that the electric activity at higher $k$ 's is more intense than the magnetic one.

The concentration of current in sheetlike structures, observed in Fig. 1, suggests that also kinetic effects may nuzzle locally as well. To get more insight in this intriguing phenomenon, we will quantify kinetic effects looking directly at the high-order velocity moments of the DF.
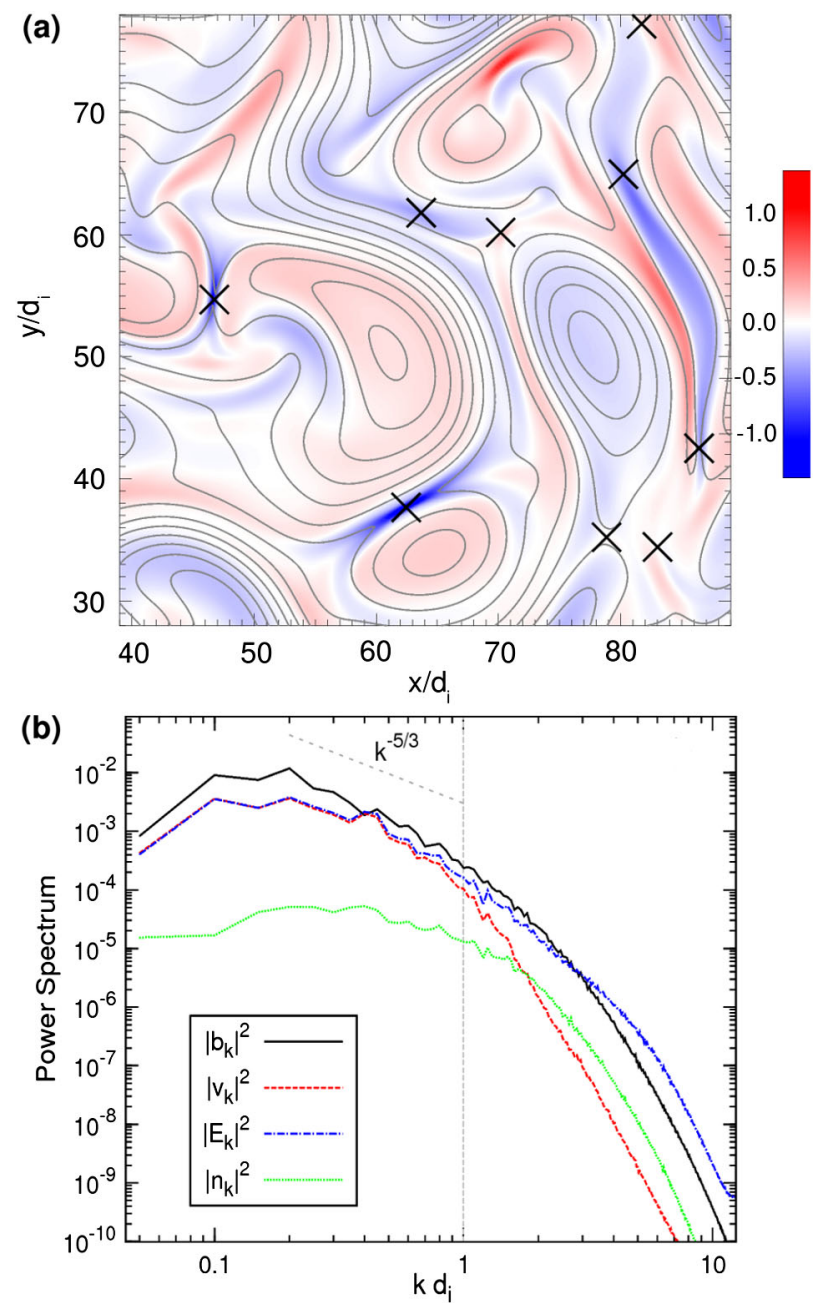

FIG. 1 (color online). (a) Shaded contours (zoom) of $j_{z}$ together with $a_{z}$ (isolines) and its $X$ points (black crosses). (b) Power spectra of ion density (green dotted), ion bulk velocity (red dashed), magnetic field (black solid), and electric field (dotdashed blue). The Kolmogorov expectation $k^{-5 / 3}$ (gray dashed) is reported as a reference, while the vertical dashed line represents the ion skin depth wave number. 
In particular, we will concentrate on the temperature and the kurtosis of $f$, that, for a Maxwellian, must be 1 and 3, respectively. The preferred directions of $f$ in the velocity space, for each $\boldsymbol{x}$, can be obtained from the stress tensor

$$
A_{i j}(\boldsymbol{x})=\frac{1}{n} \int\left(v_{i}-\left\langle v_{i}\right\rangle\right)\left(v_{j}-\left\langle v_{j}\right\rangle\right) f d^{3} v .
$$

This tensor can be studied in a diagonal form computing its eigenvalues $\left\{\lambda_{1}, \lambda_{2}, \lambda_{3}\right\}$. The respective normalized eigenvectors $\left\{\hat{\boldsymbol{e}}_{1}, \hat{\boldsymbol{e}}_{2}, \hat{\boldsymbol{e}}_{3}\right\}$ represent a proper reference frame, namely, the minimum variance frame (MVF) [17]. Note that $\lambda_{i}$ are the temperatures (for convention we choose $\lambda_{1}>\lambda_{2}>\lambda_{3}$ ) and $\hat{e}_{i}$ the anisotropy directions. For a Maxwellian, the tensor in Eq. (2) is diagonal and degenerate ( $\lambda_{i}=1$ and no preferred direction). Using the eigensystem, the temperature anisotropy is given by $\lambda_{1} / \lambda_{3}$. The probability distribution functions (PDF) of $\lambda_{1} / \lambda_{3}$ in Fig. 2(a), evaluated sampling over the entire domain of the simulation at $\tau^{\star}$ (see Table I), show that $f$ is mostly isotropic, while only few events manifest strong anisotropy $\left(\lambda_{1} / \lambda_{3} \sim 1.7\right)$. A comparison between the simulations reveals that higher level of turbulence (Runs II and III) produces patches with higher anisotropy. Moreover, also the system size influences the anisotropy phenomenonsmaller systems (Run III) are slightly more anisotropic. The latter is due to the fact that kinetic effects are more active when the system size is comparable to $d_{i}$. We would like to point out that the main ingredient that enhances anisotropy is turbulence.

The anisotropy, whose shaded contour is represented in Fig. 2(b), is confined in sheetlike structures (with the size
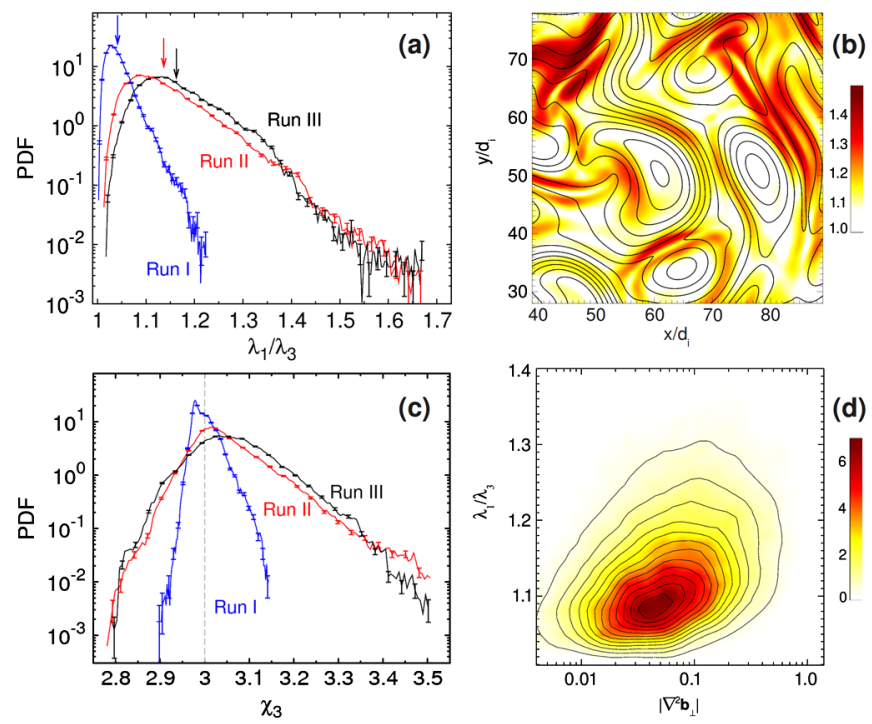

FIG. 2 (color online). (a) PDF of the temperature anisotropy $\lambda_{1} / \lambda_{3}$ for all runs (arrows represent averages); (b) shadedcontour (zoom) of the anisotropy together with the inplane magnetic field lines (black); (c) PDF of the kurtosis $\chi_{3}$; (d) joint distribution of current gradients and anisotropy $g\left(\left|\nabla^{2} b_{\perp}\right|, \lambda_{1} / \lambda_{3}\right)$. In (a) and (c) the statistical error bars are also reported. of a few $d_{i}$ ), modulated by the local magnetic field: anisotropy is low inside magnetic islands while is high in between them. These are regions of strong magnetic stress, shifted away from the $X$ points. To further investigate these kinetic effects, we inspected the normalized kurtosis (fourth-order moment):

$$
\chi_{i}(\boldsymbol{x})=\frac{\frac{1}{n} \int\left(v_{i}-\left\langle v_{i}\right\rangle\right)^{4} f d^{3} v}{\left[\frac{1}{n} \int\left(v_{i}-\left\langle v_{i}\right\rangle\right)^{2} f d^{3} v\right]^{2}} .
$$

We projected the above vector in the MVF, obtaining $\left\{\chi_{1}, \chi_{2}, \chi_{3}\right\}$. The projected kurtosis manifests opposite behavior with respect to temperatures: the strongest kurtosis is along the maximum variance frame $\hat{\boldsymbol{e}}_{3}$, namely $\chi_{3}\left(\chi_{3}\right.$ correlated with $\lambda_{1}$, not shown here.) The distributions of kurtosis manifest strong variations from Maxwellian $\left(\chi_{i}=3\right)$, suggesting that in turbulence the velocity distributions are leptokurtic [Fig. 2(c)]. Similarly to anisotropy, patterns of $\chi_{3}$ are localized in narrow layers in between magnetic vortices (not shown here). All the runs behave similarly.

The comparison between Fig. 2(b) with Fig. 1(a) suggests that these distortions are concentrated in sheetlike regions, located near the peaks of $j_{z}$. Therefore these patterns are characterized by intense $\left|\nabla^{2} \boldsymbol{b}_{\perp}\right|\left(=\left|\nabla j_{z}\right|\right)$ in a fluid model these would correspond to regions where collisional dissipation takes place. To quantify this correlation, we computed the joint PDF of current gradients and anisotropy, $g\left(\left|\nabla^{2} \boldsymbol{b}_{\perp}\right|, \lambda_{1} / \lambda_{3}\right)$, shown in Fig. 2(d). This analysis further confirms the correlation, demonstrating that kinetic effects are nonhomogeneous and concentrated in high magnetic stress regions.

It is now interesting to examine the structure of the DF in the presence of turbulence. Since Eulerian Vlasov models do not suffer from any lack of statistics in velocity space, here we provide an example of $f$, at a given $\boldsymbol{x}$. In Fig. 3(a) the isosurfaces of $f$ reveal that the DF is strongly affected by the presence of turbulence, resembling a potatolike structure elongated in the $\hat{\boldsymbol{e}}_{1}$ direction $\left(\hat{\boldsymbol{e}}_{3}\right.$ and the direction

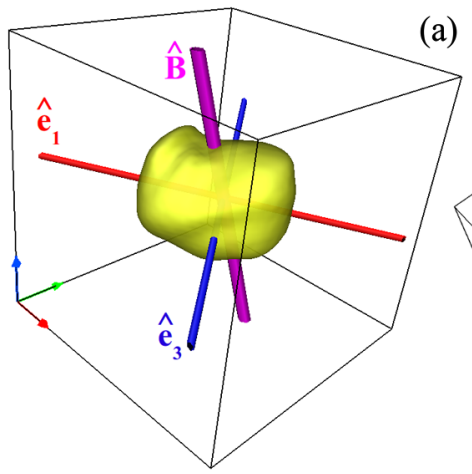

(a)

FIG. 3 (color online). (a) Isosurfaces of the velocity distribution function $f\left(\boldsymbol{x}^{\star}, \boldsymbol{v}\right)$, at a given spatial position $\boldsymbol{x}^{*} \simeq$ $(60,119) d_{i}$. (b) Two-dimensional cut of $f$ in the minimum variance frame. Thin (red) and thicker (blue) axis indicate $\hat{e}_{1}$ and $\hat{e}_{3}$, respectively. The magnetic field direction $\hat{\boldsymbol{B}}$ is represented with a thick (magenta) tube. 


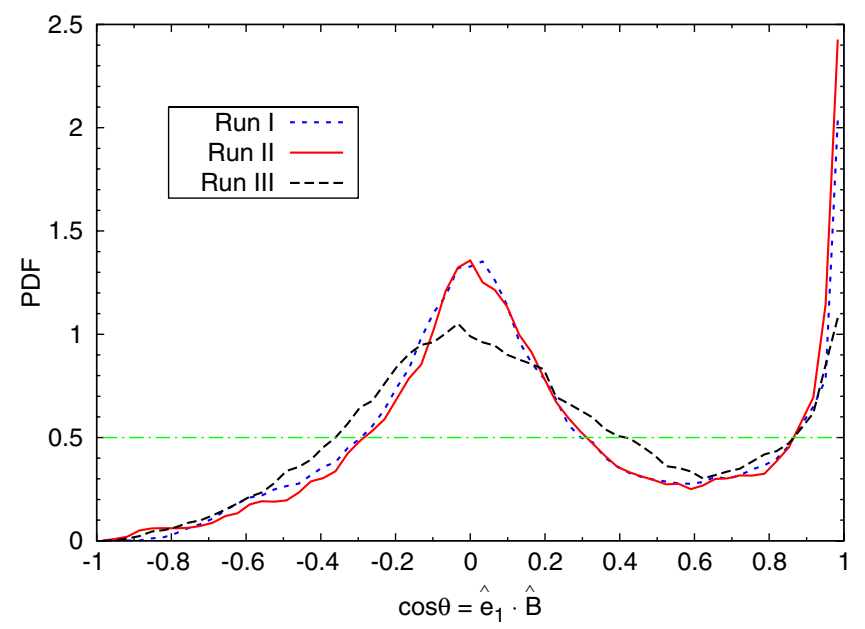

FIG. 4 (color online). PDF of the cosine angle given by Eq. (4) for all runs. The horizontal (green) dot-dashed line represents the distribution in the case of random variables.

of the local magnetic field $\hat{\boldsymbol{B}}=\boldsymbol{B} /|\boldsymbol{B}|$ are reported as well). In the same figure [panel (b)], a slice in the $\hat{\boldsymbol{e}}_{1}-\hat{\boldsymbol{e}}_{3}$ plane is reported, showing that elongation along $\hat{\boldsymbol{e}}_{1}$ is balanced by a squeezing (depression) along $\hat{\boldsymbol{e}}_{3}$. This modulation is due to the conservation of probability in phase space: the DF reacts as a balloon to the perturbations of the turbulent electromagnetic fields, producing complex deformations.

Surprisingly enough, as can be immediately noticed from Fig. 3(a), the preferred axis $\hat{\boldsymbol{e}}_{1}$ may strongly depart from the magnetic field direction $\hat{\boldsymbol{B}}$, suggesting that in a fully nonlinear regime departures from linear expectation are unavoidable. Since turbulence is a cross-scale effect, a statistical approach is required. To establish how the DF chooses its main axis, we computed, at each spatial position $\boldsymbol{x}$, the cosine angle between $\hat{\boldsymbol{e}}_{1}$ and the unit vectors of the magnetic field [18],

$$
\cos \theta(\boldsymbol{x})=\hat{\boldsymbol{e}}_{1}(\boldsymbol{x}) \cdot \hat{\boldsymbol{B}}(\boldsymbol{x}) .
$$

Note that if $\hat{\boldsymbol{e}}_{1}$ and $\hat{\boldsymbol{B}}$ were spatially random and uncorrelated, $\operatorname{PDF}(\cos \theta)$ would be a constant $\sim 0.5$. The PDF is bounded by $\cos \theta= \pm 1$. For all the runs, the PDF's of Eq. (4) are reported in Fig. 4, showing that they are not just peaked at $\cos \theta=1$. Another significant population, in fact, is present at $\cos \theta=0$ (see Fig. 3). This interesting behavior suggests that the main axis of $f$ is determined by the magnetic field in a complex way: $\hat{\boldsymbol{e}}_{1}$ can be both along or across $\boldsymbol{B}$.

To summarize, hybrid Vlasov-Maxwell simulations reveal that, in turbulence, kinetic effects manifest as snakelike patches of high anisotropy and kurtosis, nearby patterns of intense $\left|\nabla^{2} \boldsymbol{b}_{\perp}\right|$, where cyclotron and/or Landau resonances may be at work. Here, the distribution function is strongly modulated by the turbulent electromagnetic field, and elongates mainly along or across the local magnetic field. This work supports the new idea that kinetic effects in plasmas are strongly inhomogeneous, property related to the intermittent character of the magnetic field. Our results shed new light on the theory of Vlasov-Maxwell plasmas demonstrating that, when the distribution function is free to explore the entire velocity subspace, new features appear as complex interactions between the DF and the turbulent background. This statistical description of "kinetic intermittency" may challenge scientists to work on nonlinear (realistic) models of plasma dynamics, since plasma in nature is generally turbulent. Further work is needed on this path, to include important effects such as the 3D geometry, and kinetic electrons [19]. Moreover, to investigate any possible contribution of modelike fluctuations to turbulence (such as Alfvén, whistler, and kinetic-Alfvén waves), it would be important to carry out a space-time analysis in the presence of a driving [20].

We acknowledge the INAF-CINECA Key-Project 2010, the EU DEISA Extreme Computing Initiative (EU FP6 Project No. RI-031513 and FP7 Project No. RI-222919) and the Proposal No. 269297 TURBOPLASMAS.

[1] E. Marsch, Living Rev. Solar Phys. 3, 1 (2006); E. N. Parker, Astrophys. J. 330, 474 (1988).

[2] S.P. Gary, Theory of Space Plasma Microinstabilities (Cambridge University Press, Cambridge, 1993); P. Hellinger et al., Geophys. Res. Lett. 33, L09101 (2006).

[3] K. T. Osman et al., Astrophys. J. Lett. 727, L11 (2011).

[4] T. Tatsuno et al., Phys. Rev. Lett. 103, 015003 (2009).

[5] R. C. Davidson, Physics of Nonneutral Plasmas (AddisonWesley, Redwood City, CA, 1990).

[6] R. Bruno and V. Carbone, Living Rev. Solar Phys. 2, 4 (2005).

[7] F. Sahraoui et al., Phys. Rev. Lett. 102, 231102 (2009).

[8] J. V. Holloweg and P. A. Isenberg, J. Geophys. Res. 107, 1147 (2002); E. Marsch, X.Z. Ao, and C.-Y. Tu, J. Geophys. Res. 109, A04102 (2004).

[9] E. Marsch et al., J. Geophys. Res. 87, 52 (1982); C.-Y. Tu, E. Marsch, and Z.-R. Qin, J. Geophys. Res. 109, A05101 (2004).

[10] F. Valentini et al., J. Comput. Phys. 225, 753 (2007); F. Valentini, F. Califano, and P. Veltri, Phys. Rev. Lett. 104, 205002 (2010).

[11] M. Wan et al., Phys. Plasmas 17, 082308 (2010).

[12] The normalized electron skin depth, $d_{e}=\sqrt{\left(m_{e} / m_{i}\right)} \simeq$ 0.02 , cannot be resolved with the spatial resolution used here. We prefer not to use a reduced mass ratio since it would introduce artificial scale couplings.

[13] P. D. Mininni and A. Pouquet, Phys. Rev. E 80, 025401 (2009).

[14] S. Servidio et al., Phys. Rev. Lett. 102, 115003 (2009).

[15] J. F. Drake et al., Astrophys. J. 709, 963 (2010).

[16] S. D. Bale et al., Phys. Rev. Lett. 94, 215002 (2005); S. Servidio et al., Planet. Space Sci. 55, 2239 (2007).

[17] B. U. O. Sonnerup and L. J. Cahill, J. Geophys. Res. 72, 171 (1967).

[18] R. M. Kerr, Phys. Rev. Lett. 59, 783 (1987).

[19] W. Daughton et al., Nature Phys. 7, 539 (2011); S. Saito et al., Phys. Plasmas 15, 102305 (2008).

[20] T. N. Parashar et al., Phys. Plasmas 17, 102304 (2010). 